GRP-146 PRACTICE ASSESSMENT OF ANTIBIOTHERAPY GUIDELINES FOR URINARY INFECTIONS AND LOWER RESPIRATORY TRACT INFECTIONS IN ELDERLY PATIENTS OF A NURSING HOME

doi:10.1136/ejhpharm-2013-000276.146

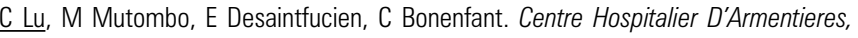
Nord, Armentières Cedex, France

Background The elderly population keeps growing and a lot are living in nursing homes, where infections are frequent as patients are weak and the risk of transmission is high.

Purpose Nursing home physicians wanted to standardise practise for antibiotic treatment. With the aim of quality and safety of care, prescriptions for antibiotics for urinary infections (UIs) and lower respiratory tract infections (LRTIs) were assessed.

Materials and Methods A prospective study: for two months, each prescription for antibiotics was studied. For each antibiotic, the site of infection, dose, duration and reassessment of the treatment after 48 to 72 hours were checked. These criteria were compared to guidelines approved by our 'antibiotics committee'. Results of bacteriological samples, history of antibiotic treatment in the previous three months were also checked.

Results 82 patients were treated with antibiotics. Mean age was 81.4 years old. There were 56 LRTIs, 13 UIs. There are no guidelines for the treatment of bronchitis in the elderly so assessment of antibiotic treatment was not possible. The choice of drug was appropriate in $100 \%$ of UIs and LRTIs. The dose was adequate in 100\% of the cases. Duration of treatment was adequate in $50 \%$ for cystitis, $0 \%$ for prostatitis, and $97.4 \%$ for LRTIs. Most of treatment was empirical (95.5\%), few bacteriological samples were taken: $3 \%$ for pneumonia, $7.10 \%$ for bronchitis, $0 \%$ for exacerbations of chronic obstructive pulmonary disease, $62.5 \%$ for cystitis, and $33.3 \%$ for prostatitis. Traceability of reassessment after 48-72 hours couldn't be found in $98.7 \%$ of cases.

Conclusions Specific guidelines for antibiotic treatment were written to facilitate and standardise the prescribing process. Pharmacists and physicians decided to treat bronchitis like pneumonia. Indeed, this study underlined the specificity of patient care in nursing homes. However, more bacteriological samples need to be taken to prescribe the right treatment and to prevent antibiotic resistance.

No conflict of interest.

\section{GRP-147 PRE-POST STUDY OF INTERRUPTIONS IN A PHARMACY DEPARTMENT}

doi:10.1136/ejhpharm-2013-000276.147

A Guérin, E Caron, D Lebel, JF Bussières. CHU Sainte-Justine, Pharmacy, Montreal, Canada

Background Interruptions are a major concern in hospital pharmacy settings, given the nature and the requirements of the work such as sustained focused attention, validating prescriptions and performing complex processes. Interruptions may jeopardise the safe delivery of pharmaceutical services.

Purpose The primary objective was to compare the number of stimuli per hour received and made by pharmacists and pharmacy technicians between 2010 and 2012. The secondary objective was to evaluate the impact of five corrective measures.

Materials and Methods This was a pre-post cross-sectional observational study. The study was conducted in the main dispensing area of the pharmacy department of a University Hospital Center. The area is composed of three data entry stations each assigned one pharmacist and one pharmacy technician. Stimuli received and made by pharmacists and pharmacy technicians were counted before (2010) and after (2012) the implementation of corrective measures. The effect of five corrective measures was measured with a t-test for targeted stimuli.

Results Sixty-two random 30-minute observation periods in 2010 ( $\mathrm{n}=2,663$ stimuli) and 31 periods in 2012 ( $\mathrm{n}=1,217$ stimuli) were conducted. An average rate of $85.9 \pm 22.2$ stimuli per hour was calculated in 2010 vs. $78.52 \pm 20.1$ in $2012(P=0.06)$. We observed a statistically significant decrease in the mean rate of stimuli per hour for three types of stimulus for pharmacists (i.e. printer noise $3.7 \pm 2.4$ vs. $0.6 \pm 1.8 p<0.001$, face-to-face non-professional conversations $4.4 \pm 4.2$ vs. $1.2 \pm 1.8 p=0.003$, Web browsing $1.3 \pm 2.2$ vs. $0 \pm 0 \mathrm{p}=0.009)$ and for one type of stimuli for pharmacy technicians (i.e. printer noise $4.7 \pm 3.2$ vs. $0.75 \pm 1.8 p<0.001$ ).

Conclusions Despite the corrective measures, there was no statistically significant difference between the rates of stimuli per hour observed in 2010 and 2012. Other studies are needed to identify more efficient corrective measures and to better describe the nature and the impact of stimuli, distractions and interruptions in pharmacy practise.

No conflict of interest.

\section{GRP-148 PREPARATION OF A RISK MAP FOR A PHARMACY SERVICE IN A HEALTHCARE AREA}

doi:10.1136/ejhpharm-2013-000276.148

'MA Almendral Vicente, 1 'R Gavira Moreno, 'P Gómez Germá, 'MT Gómez de Travecedo y Calvo, ${ }^{2} \mathrm{~S}$ López Rubio, ${ }^{2} \mathrm{C}$ Escudero Castillo, ${ }^{2} \mathrm{JC}$ Morales Serna, ${ }^{2} \mathrm{MD}$ Pérez Pacheco, ${ }^{2}$ A García Bonilla, ${ }^{1}$ G González Rosa. 'Hospital del SAS de Jerez, Farmacia, Jerez de la Frontera (Cádiz), Spain; ${ }^{2}$ Atención Primaria de Jerez, Farmacia, Jerez de la Frontera (Cádiz), Spain

Background Safety is a priority in the medication use process. Purpose To prepare a risk map to identify the risks to safety in a Pharmacy Service and prioritise the risks found.

Materials and Methods Formation of a multidisciplinary working group.

1. Description of the different processes linked to the healthcare area which included the clinical pharmacy unit sited in the Hospital and primary health care pharmacies.

2. Identify the risks linked to the processes by the FMEA or Failure Mode and Effect Analysis: (mode of failure, cause of failure, effect of failure)

3. Rank risks by a risk matrix (vertical axis: likelihood and horizontal axis: consequence). The matrix uses a colour code.

4. Prioritize the risks by the modified Hanlon method. Once the risks have been identified, rate each risk on the following criteria: size of the risk (A), magnitude of the risk (B), feasibility of possible interventions (C) and effectiveness of potential interventions $(D)$. Then, calculate priority scores using the following formula: $(\mathrm{A}+\mathrm{B}) \mathrm{C} \times \mathrm{D}$.

Results In the unit clinical hospital pharmacy, eleven processes were identified: 1) Drug acquisitions; 2) Pharmacy stores; 3) Drug stock management; 4) Automatic dispensing systems; 5) Traditional drugdispensing systems; 6) Out-patient pharmacy; 7) Patient education; 8) Centralized cytotoxic preparation; 9) Pharmacy production; 10) Intravenous admixture preparation; 11) Clinical trials. The process with the highest risk score was the centralised cytotoxic preparation.

In primary health care pharmacies, eight processes were identified: 1) Methadone treatment programme; 2) Drug stock management; 3) Pharmacy stores; 4) Vaccination dispensing and storage; 5) Management of off-label drug use; 6) Drug prescription validation; 7) Health education; 8) Rational use of drugs. The process with the highest risk score was vaccination storage and dispensing. Conclusions Preparing a risks map is a useful tool to identify risks to safety. Prioritization of the risks allows us to identify the most unsafe practises and provides a starting point for implementing measures to improve safety in the work environment.

No conflict of interest. 